Return to the Manage Active Submissions page at http://spie.org/submissions/tasks.aspx and approve or disapprove this submission. Your manuscript will not be published without this approval. Please contact author_help@spie.org with any questions or concerns.

\title{
Raman based power combining and wavelength conversion of high power ytterbium fiber lasers \\ Santosh Aparanji ${ }^{\mathrm{a}}$, V. Balaswamy ${ }^{\mathrm{a}}$, S. Arun ${ }^{\mathrm{a}}$, V. R. Supradeepa*a \\ ${ }^{a}$ Centre for Nano Science and Engineering, Indian Institute of Science, Mathikere, Bangalore, Karnataka, India 560012
}

\begin{abstract}
In this work, we demonstrate an architecture to perform Raman-based power combining and simultaneous wavelength conversion of two independently controlled high-power Ytterbium doped fiber lasers operating at different wavelengths into a single laser line at the 1.5-micron band. Specifically, we have been able to achieve an in-band output power of $\sim 99 \mathrm{~W}$ with a conversion of $\sim 64 \%$ of the quantum limited efficiency. This power combining is illustrated for different cases of the input wavelengths of the Ytterbium fiber laser. In each case, we have been able to demonstrate a power combining of $>87 \mathrm{~W}$ in the final 1.5 -micron band, with more than $85 \%$ of the fraction of the power residing in the final desired band.
\end{abstract}

Keywords: Lasers; Lasers, fiber; Lasers, ytterbium; Scattering, Raman; Lasers, Raman; Laser beam combining; Lasers, distributed-feedback; Nonlinear wave mixing.

\section{INTRODUCTION}

Power scaling in fiber lasers has mostly been confined to the Ytterbium emission band of $1.06 \mathrm{um}$, owing to Ytterbium's desirable properties of low quantum defect and ability to support enhanced doping concentrations ${ }^{1}$. However, desirable attributes of eye-safety and atmospheric transparency are lacking in the $\mathrm{Yb}$ emission band, but exist in other bands such as the 1.5-micron band, where Erbium-doped or Erbium-Ytterbium co-doped fiber lasers are most suited to operate ${ }^{2}$. But power-scaling in Erbium is not without associated problems of quenching, parasitic lasing, ion-pair formation and reduced mode quality ${ }^{1}$. These drawbacks can be mitigated by using Cascaded Raman Fiber lasers, and they are an attractive option of generating high power in the 1.5-micron band. Several architectures for efficiently Raman converting a $\mathrm{Yb}$ laser input to the desired wavelength band have been proposed, where a single high-power $\mathrm{Yb}$ laser source is Stokes shifted to the desired band ${ }^{2}$. Were one to indefinitely scale the power of the high-power $\mathrm{Yb}$ source, one would have to grapple with the problem of undue stress in the laser components, and ultimately would be limited by the onset of instabilities due to the coupling of the Raman conversion module with the rare-earth doped stage ${ }^{3,4}$. One way to deal with this is to power-combine multiple, lower-power modules to achieve power-scaling such as coherent combining in free space. However, such architectures have so far been complex, and it is desirable to have a simpler architecture for this purpose. In this work, we propose a novel architecture which simultaneously achieves both power combining and wavelength conversion of 2 independently-controlled $\mathrm{Yb}$ lasers operating at different wavelengths into a single laser laser at 1.5 micron band.

\section{EXPERIMENTAL SETUP}

Fig. 1 shows the conceptual illustration of nonlinear power combining of two independently controlled, high-power Ytterbium-doped fiber lasers operating at two different wavelengths. The two independent laser outputs are wavelengthcombined by the wavelength-combiner and then sent to a Raman conversion stage which serves to convert the input through a series of Stokes shifts to one final laser line in the desired wavelength band in the process. In this work, we propose a novel architecture which simultaneously achieves both power combining and wavelength conversion of two independently-controlled Ytterbium-doped fiber lasers operating at different wavelengths into a single laser laser at 1.5 micron band. 
Return to the Manage Active Submissions page at http://spie.org/submissions/tasks.aspx and approve or disapprove this submission. Your manuscript will not be published without this approval. Please contact author_help@spie.org with any questions or concerns.

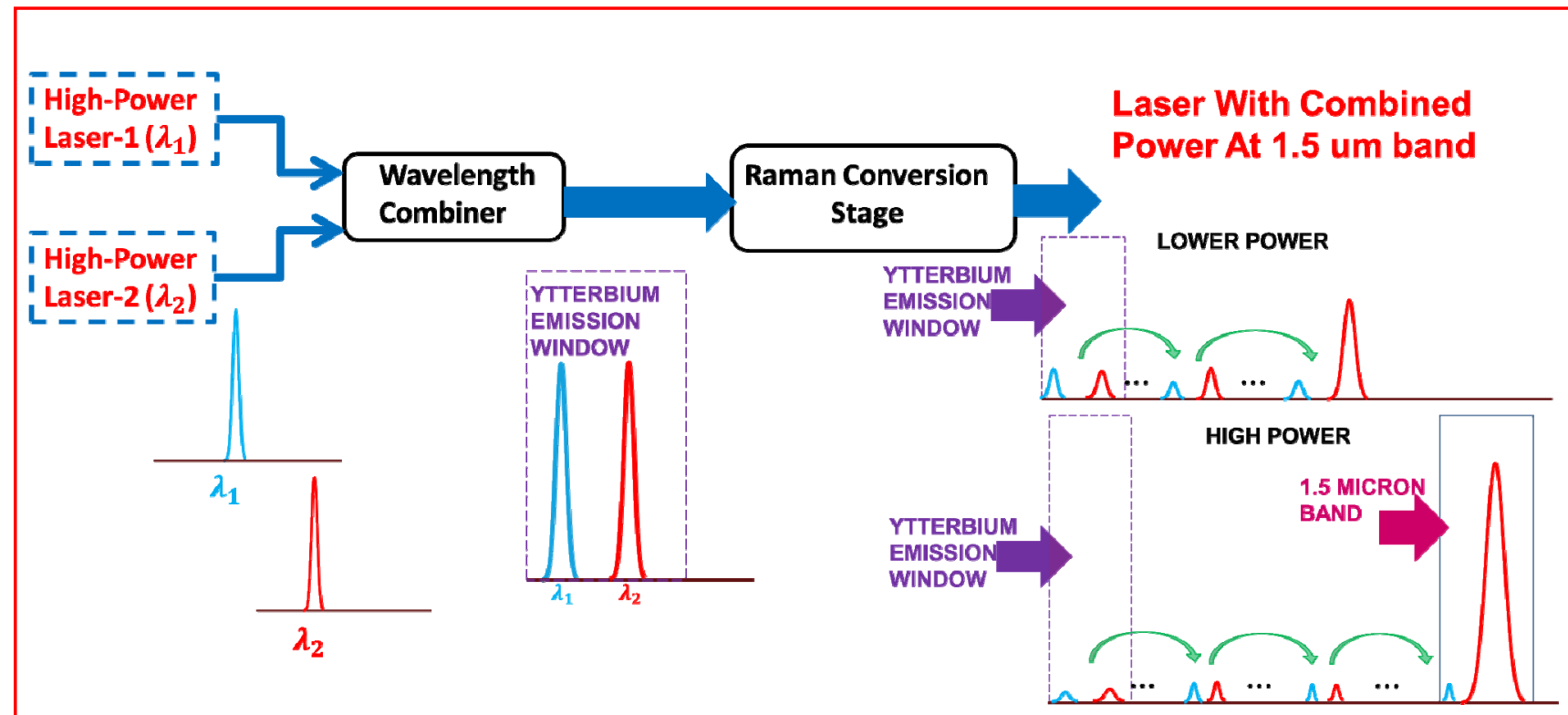

Figure 1. Conceptual schematic of a nonlinear Raman power combining and wavelength conversion.

Fig. 2 depicts the architecture implementing such a schematic, specifically ensuring that the two lasers operating at 1117 $\mathrm{nm}$ and $1075 \mathrm{~nm}$ are not separated by one Stokes' shift to demonstrate the general nature of this technique. A case where the 2 lasers are indeed separated by a Stokes' shift is also presented hereinafter. The Raman filter fiber used has an intrinsic cutoff at $1500 \mathrm{~nm}$ and ensures the termination of the cascaded Raman conversion. The concept of distributed Raman feedback was recently used to eliminate gratings in Raman lasers and this approach is adopted here by the use of the feedback coupler in this architecture ${ }^{5,6}$. In this architecture, the feedback coupler serves to provide the seeding for all the intermediate Stokes so as to make the process of stimulated Raman scattering in the forward direction highly efficient.

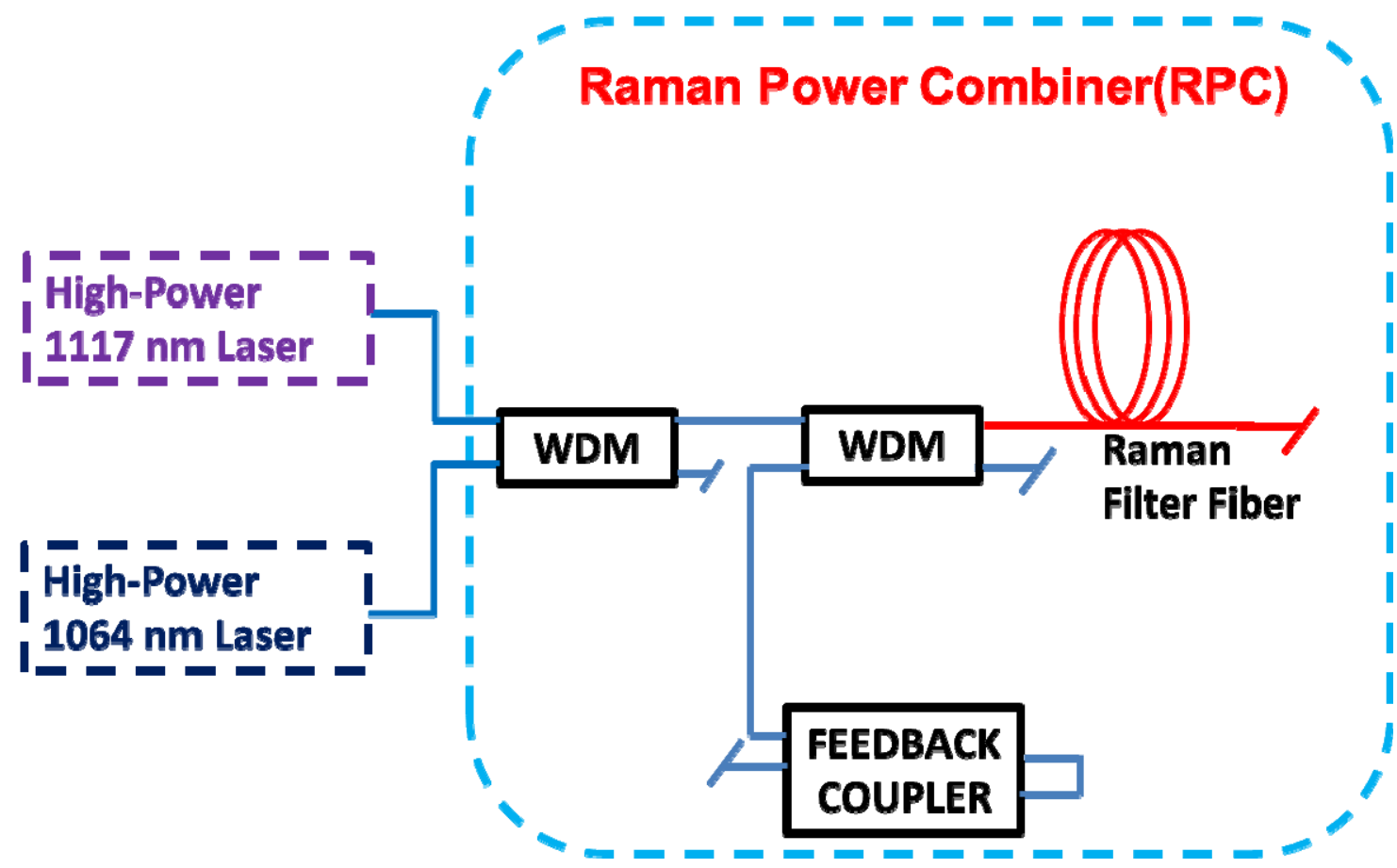

Figure 2. Architecture implementing nonlinear Raman power combining using two independently controlled Ytterbium doped fiber lasers operating at $1117 \mathrm{~nm}$ and $1075 \mathrm{~nm}$. 
Return to the Manage Active Submissions page at http://spie.org/submissions/tasks.aspx and approve or disapprove this submission. Your manuscript will not be published without this approval. Please contact author_help@spie.org with any questions or concerns.

\section{RESULTS}

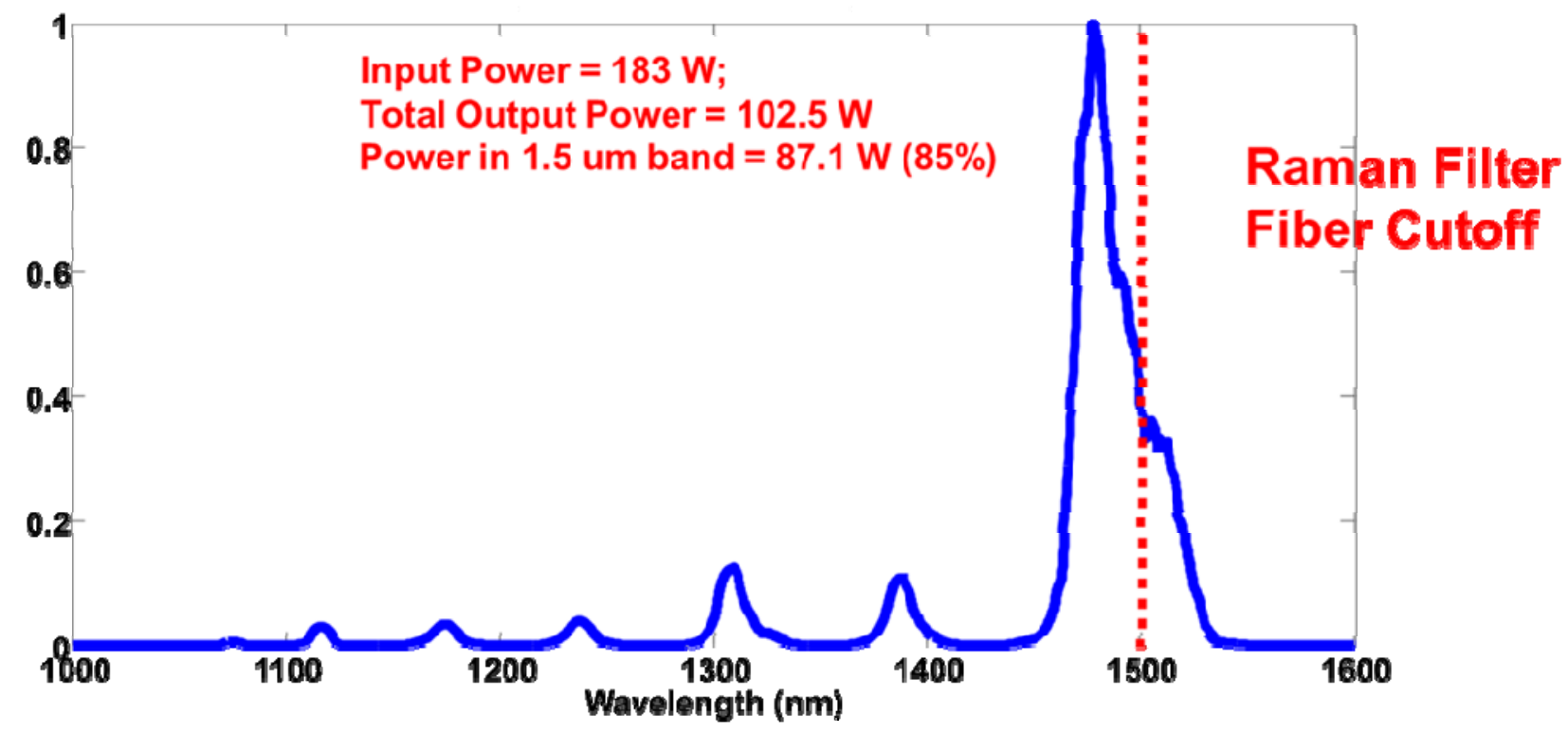

Figure 3. Architecture implementing nonlinear Raman power combining using two independently controlled Ytterbium doped fiber lasers operating at $1117 \mathrm{~nm}$ and $1075 \mathrm{~nm}$.

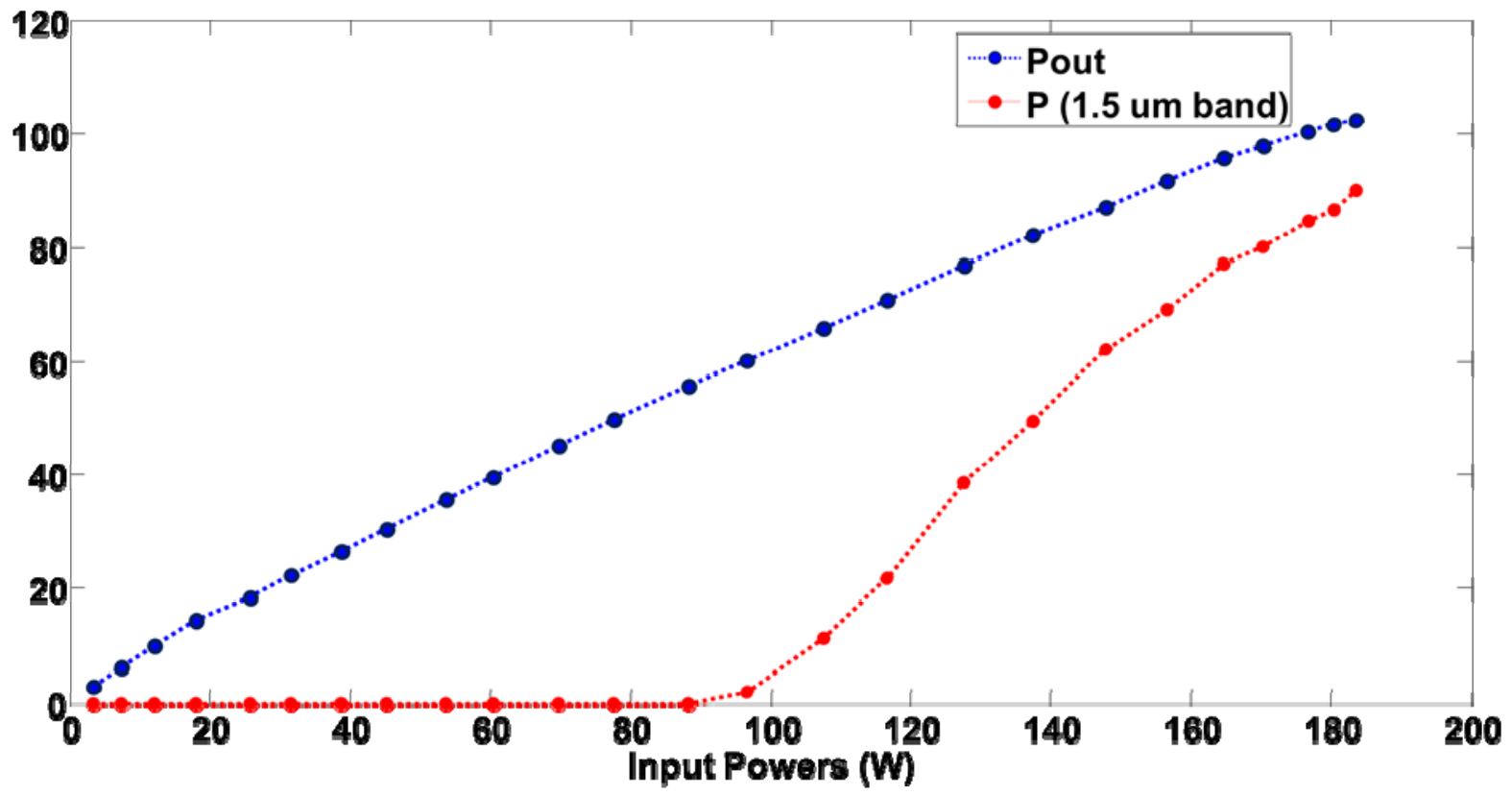

Figure 4. Evolution of the total output power and the power in the 1.5-micron band as a function of the total laser input power with the input laser wavelengths at $1117 \mathrm{~nm}$ and $1075 \mathrm{~nm}$.

Fig. 3 shows the final spectrum at a full power of $183.4 \mathrm{~W}$ from the two independent lasers, where one of the lasers is at $1117 \mathrm{~nm}$ and the other is at $1075 \mathrm{~nm}$. It can be seen from this spectrum that most of the output power now resides in the 1.5-micron band with residual powers in all the intermediate Stokes wavelengths. The presence of the Raman filter fiber 
Return to the Manage Active Submissions page at http://spie.org/submissions/tasks.aspx and approve or disapprove this submission. Your manuscript will not be published without this approval. Please contact author_help@spie.org with any questions or concerns.

has ensured that the spectrum doesn't evolve beyond the 1.5 -micron band. The power in the 1.5 -micron band is $87.1 \mathrm{~W}$ for a total output power of $102.5 \mathrm{~W}$, achieving a high $85 \%$ conversion. For the combined input laser power of $183 \mathrm{~W}$, this corresponds to $\sim 64 \%$ of the quantum limited efficiency. A plot of the total recorded output power from the Raman Power Combiner (RPC) and the power in the 1.5-micron band as a function of the combined laser input power is shown in Fig. 4. It can be seen that with increasing input laser powers, there is a greater degree of conversion to the final 1.5micron band.

In order to show the versatility of this nonlinear power-combining technique we have performed the experiment with the input lasers operating at different wavelengths. Specifically, the $1117 \mathrm{~nm}$ laser is held fixed, and the other input laser is varied in wavelength to operate at $1070 \mathrm{~nm}$ and $1064 \mathrm{~nm}$.

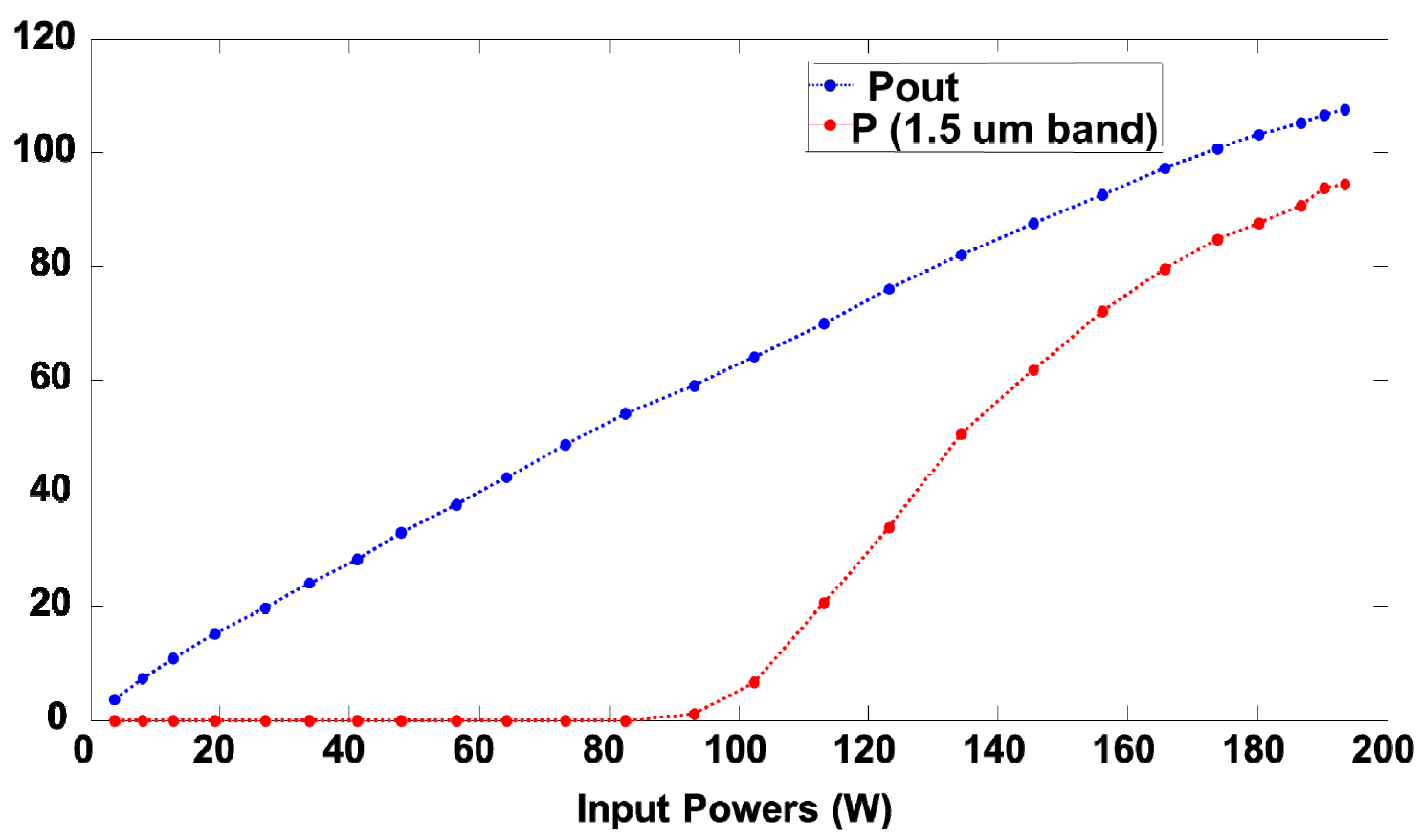

Figure 5. Evolution of the total output power and the power in the 1.5-micron band as a function of the total laser input power with the input laser wavelengths operating at $1117 \mathrm{~nm}$ and $1070 \mathrm{~nm}$.

Fig. 5 shows the evolution of the total output power and the power in the final 1.5 -micron band as a function of the combined input laser power when the two input lasers are operating at wavelengths of $1117 \mathrm{~nm}$ and $1070 \mathrm{~nm}$. It can be seen in this case as well, that with greater input powers, there is a greater degree of conversion to the final 1.5-micron band. In this case, at full power for a combined input power of $193.4 \mathrm{~W}$, the power in the 1.5 -micron band is $94.3 \mathrm{~W}$ for a total output power of around 108 W. Fig. 6 illustrates this evolution for the special case where the two input lasers are separated by one Stokes shift, viz., the two input lasers operate at $1117 \mathrm{~nm}$ and $1064 \mathrm{~nm}$. It can be seen that even in this special scenario, the nonlinear power combining technique works to provide a simultaneous wavelength conversion and power combining in the final 1.5-micron band. Specifically, for a total input laser power of $201 \mathrm{~W}$, the power in the 1.5micron band is an impressive $99 \mathrm{~W}$ for a total output power corresponding to $112 \mathrm{~W}$. 
Return to the Manage Active Submissions page at http://spie.org/submissions/tasks.aspx and approve or disapprove this submission. Your manuscript will not be published without this approval. Please contact author_help@spie.org with any questions or concerns.

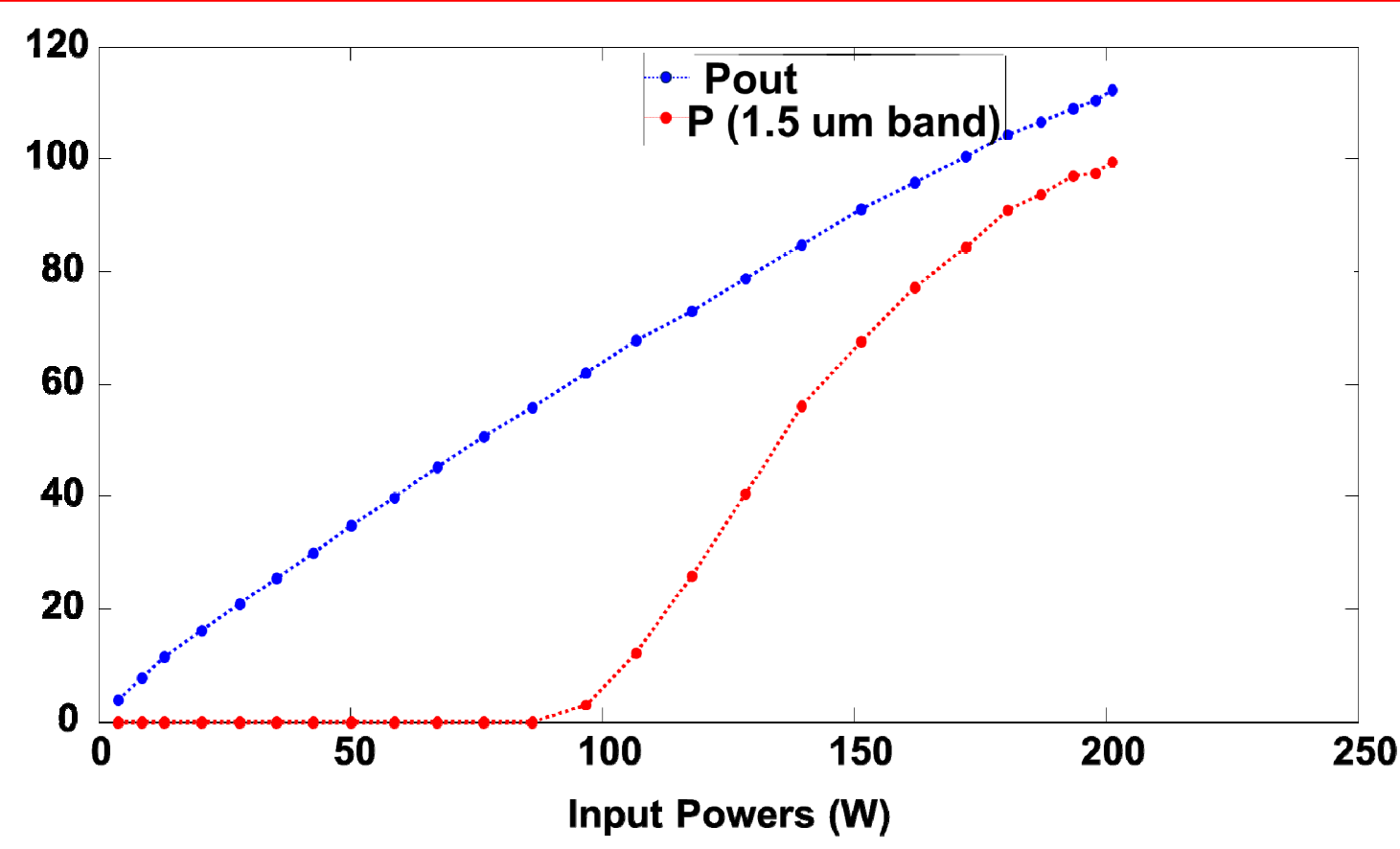

Figure 6. Evolution of the total output power and the power in the 1.5-micron band as a function of the total laser input power with the input laser wavelengths operating at $1117 \mathrm{~nm}$ and $1064 \mathrm{~nm}$.

(a)

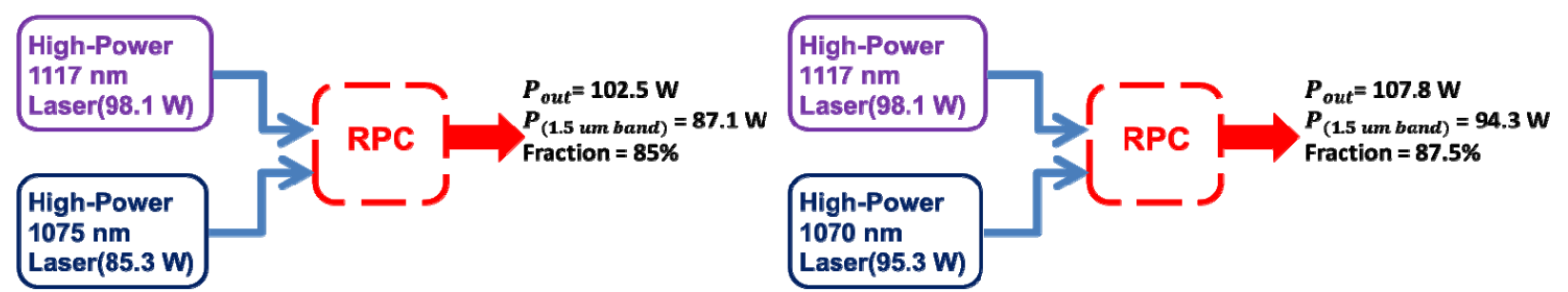

(c)

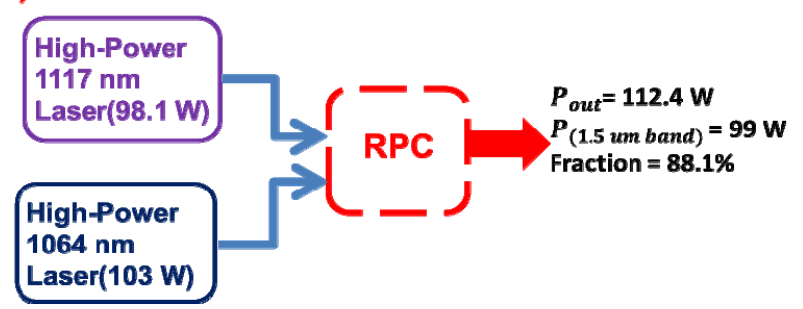

Figure 7. Schematic representation of the results of the nonlinear Raman-based power combining for the case of the 3 different input wavelength combinations, with one laser input held at $1117 \mathrm{~nm}$ and the other input at (a) $1075 \mathrm{~nm}$; (b) 1070 $\mathrm{nm}$; (c) $1064 \mathrm{~nm}$.

Fig. 7 summarizes the results obtained for the nonlinear Raman-based power combining technique with different combinations of the input laser wavelengths. It can be seen from Fig. 7(b) and Fig. 7(c) that even for the case where the input laser operates at $1070 \mathrm{~nm}$ and $1064 \mathrm{~nm}$ respectively, the power in the final 1.5 -micron band at full power is $>90$ $\mathrm{W}$, and the fraction of the total output power in this band is more than $87 \%$. In both cases for a combined input laser 
Return to the Manage Active Submissions page at http://spie.org/submissions/tasks.aspx and approve or disapprove this submission. Your manuscript will not be published without this approval. Please contact author_help@spie.org with any questions or concerns.

power of $193.4 \mathrm{~W}$ and $201 \mathrm{~W}$, this corresponds to $\sim 64 \%$ of the quantum limited efficiency from $1117 \mathrm{~nm}$ to the final 1.5 -micron band. This shows the true versatility of this technique to achieve simultaneous power combining and wavelength conversion to a single lasing line. This technique is easily scalable and currently, the output powers are only limited by available powers from the input lasers.

\section{REFERENCES}

[1] Richardson, D. J., Nilsson, J. and Clarkson, W. A., "High power fiber lasers: current status and future perspectives [Invited]," J. Opt. Soc. Am. B 27, B63-B92 (2010).

[2] Supradeepa, V. R., Feng, Y. and Nicholson, J. W., "Raman fiber lasers." Journal of Optics 19.2: 023001 (2017).

[3] Nicholson, J. W., Yan, M. F., Wisk, P., Fleming, J., DiMarcello, F., Monberg, E., Taunay, T., Headley, C. and DiGiovanni, D. J., "Raman fiber laser with $81 \mathrm{~W}$ output power at $1480 \mathrm{~nm}$," Opt. Lett. 35, 3069-3071 (2010).

[4] Aparanji, S., Balaswamy, V. and Supradeepa, V. R., "On the Stability of Raman Fiber Lasers," 13th Int. Conf. on Fiber Opt. Phot., OSA Technical Digest (online), paper Th3A.20 (2016).

[5] Babin, S. A., Zlobina, E. A., Kablukov, S. I. and Podivilov, E. V., "High-order random Raman lasing in a PM fiber with ultimate efficiency and narrow bandwidth," Sci. Rep. 6, 22625 (2016).

[6] Arun, S., Balaswamy, V., Aparanji, S. and Supradeepa, V. R., "High Power, Grating-Free, Cascaded Raman Fiber Lasers" Conference on Lasers and Electro-optics (CLEO) Europe, CJ-2.4, (2017). 